\title{
Correction: A Smartphone App to Improve Medication Adherence in Patients With Type 2 Diabetes in Asia: Feasibility Randomized Controlled Trial
}

\author{
Zhilian Huang ${ }^{1,2}$, MPH; Eberta Tan ${ }^{3}$, MRCP (UK), MMed, MBBS; Elaine Lum ${ }^{1,4,5}$, PhD, MClinPharm, BPharm; \\ Peter Sloot ${ }^{6,7,8}$, PhD; Bernhard Otto Boehm ${ }^{9,10}$, FRCP, MD; Josip Car ${ }^{1}$, FRCP, FFPH, MSc, PhD, MD \\ ${ }^{1}$ Centre for Population Health Sciences, Lee Kong Chian School of Medicine, Nanyang Technological University, Singapore, Singapore \\ ${ }^{2}$ NTU Institute for Health Technologies, Interdisciplinary Graduate School, Nanyang Technological University, Singapore, Singapore \\ ${ }^{3}$ Department of Endocrinology, Changi General Hospital, Singapore, Singapore \\ ${ }^{4}$ Institute of Health and Biomedical Innovation, Queensland University of Technology, Queensland, Australia \\ ${ }^{5}$ School of Clinical Sciences, Faculty of Health, Queensland University of Technology, Queensland, Australia \\ ${ }^{6}$ Complexity Institute, Nanyang Technological University, Singapore, Singapore \\ ${ }^{7}$ Institute for Advanced Science, University of Amsterdam, Amsterdam, Netherlands \\ ${ }^{8}$ ITMO University, Saint Petersburg, Russian Federation \\ ${ }^{9}$ Lee Kong Chian School of Medicine, Nanyang Technological University, Singapore, Singapore \\ ${ }^{10}$ Department of Endocrinology, Tan Tock Seng Hospital, Singapore, Singapore
}

\section{Corresponding Author:}

Zhilian Huang, MPH

Centre for Population Health Sciences

Lee Kong Chian School of Medicine

Nanyang Technological University

Clinical Sciences Building, Level 18

11 Mandalay Road

Singapore, 308232

Singapore

Phone: 6565141221

Email: zhuang014@e.ntu.edu.sg

\section{Related Article:}

Correction of: https://mhealth.jmir.org/2019/9/e14914/

(JMIR Mhealth Uhealth 2020;8(4):e18411) doi: 10.2196/18411

The authors of "A Smartphone App to Improve Medication Adherence in Patients With Type 2 Diabetes in Asia: Feasibility Randomized Controlled Trial" (JMIR Mhealth Uhealth 2019;7(9):e14914) noticed an error in the Results section of their published article. Under the "Adherence to Trial Participation" subsection of the Results section, the following sentence:

Eight participants had $100 \%$ adherence for the first 2 weeks of the intervention, which was decreased to $4 \%$ by the third week of the intervention.

Has been changed to:
Eight participants had $100 \%$ adherence for the first 2 weeks of the intervention, which was decreased to four participants by the third week of the intervention.

This change does not affect any of the data presented in the Results section of the paper.

The correction will appear in the online version of the paper on the JMIR website on April 29, 2020, together with the publication of this correction notice. Because this was made after submission to PubMed, PubMed Central, and other full-text repositories, the corrected article has also been resubmitted to those repositories. 
This is a non-peer-reviewed article. Submitted 25.02.20; accepted 25.02.20; published 29.04.20.

Please cite as:

Huang Z, Tan E, Lum E, Sloot P, Boehm BO, Car J

Correction: A Smartphone App to Improve Medication Adherence in Patients With Type 2 Diabetes in Asia: Feasibility Randomized Controlled Trial

JMIR Mhealth Uhealth 2020;8(4):e18411

URL: https://mhealth.jmir.org/2020/4/e18411

doi: $10.2196 / 18411$

PMID: $\underline{32348282}$

CZhilian Huang, Eberta Tan, Elaine Lum, Peter Sloot, Bernhard Otto Boehm, Josip Car. Originally published in JMIR mHealth and uHealth (http://mhealth.jmir.org), 29.04.2020. This is an open-access article distributed under the terms of the Creative Commons Attribution License (https://creativecommons.org/licenses/by/4.0/), which permits unrestricted use, distribution, and reproduction in any medium, provided the original work, first published in JMIR mHealth and uHealth, is properly cited. The complete bibliographic information, a link to the original publication on http://mhealth.jmir.org/, as well as this copyright and license information must be included. 